\title{
Investigation of human papillomavirus prevalence in married women and molecular characterization and phylogenetic analysis of the virus
}

\author{
Ayse Erdem Yayla', Berrin Goktug Kadioglu², Ayse Aydin ${ }^{3}$ Osman Aktas ${ }^{1}$ \\ ${ }^{1}$ Department of Medical Microbiology, School of Medicine, Ataturk University; ${ }^{2}$ Department of Gynecology and Obstetrics, Health Sciences University, \\ Erzurum Regional Training and Research Hospital; ${ }^{3}$ Department of Birth, Women's Health and Diseases Nursing, Faculty of Nursing, Ataturk University, \\ Erzurum, Turkey
}

\section{Objective}

Human papillomaviruses (HPVs) are among the agents responsible for infection and cancer of the skin and mucous membranes in the human body. The aim of this study was to investigate the frequency and type distribution of HPVs in married female patients with gynecological complaints, who had visited the Maternity Hospital in Erzurum, Turkey.

\section{Methods}

In this study, 263 cervical swab samples were taken from married women using the Pap smear method and were investigated for positive reactivity against HPV. The L1 gene region of HPV was investigated using molecular methods. For this purpose, polymerase chain reaction (PCR) assays and sequence analysis of positive samples were performed. Phylogenetic analyses were performed using a bioinformatics approach after sequencing.

\section{Results}

HPV-DNA was detected in $17(6.5 \%)$ samples. Highest positive reactivity to HPV-DNA was found in the 35-44 age group at $9.2 \%$. Fourteen out of seventeen positive samples were included in the phylogenetic analysis. All isolates clustered in the Alphapapillomavirus genus. Six samples were found to be HPV 70 positive, four were HPV 16 positive, and the rest were HPV 54, 72, 81, and 114 positive. When genotyping data were evaluated according to the risk group, we found that $28.6 \%$ of the 14 samples were found to be high risk-HPV, and $71.4 \%$ were low risk-HPV.

\section{Conclusion}

As per our knowledge, this is the first report on the phylogenetic analysis of HPV genotypes isolated from women in Turkey. The prevalence of low- and-high risk HPV was determined in married women in Erzurum, and these results contribute to the epidemiological data on the distribution of HPV types for this region.

Keywords: Papillomavirus infections; Cervical smears; Polymerase chain reaction; Turkey

\section{Introduction}

Persistent infection with the human papilloma virus (HPV) can cause malignant changes in the genital areas of men and women [1]. Human papilloma viruses (HPVs), responsible for causing cervical cancers, which affect a large number of individuals of the younger generation, also cause cancers of the vagina, vulva, penis, and anus, as well as infections of the head and neck region, conjunctiva, ear canals, nasal sinuses, and oral cavities [2]. After breast cancer, cervical cancer is the second most common cancer in the world among women, with a higher frequency of incidence and rate of mortality in
Received: 2018.07.29. Revised: 2018.12.29. Accepted: 2019.01.27. Corresponding author: Osman Aktas

Department of Medical Microbiology, School of Medicine, Atatürk University, Erzurum 25240, Turkey

E-mail: osaktas@atauni.edu.tr

https://orcid.org/0000-0002-7762-4108

Articles published in Obstet Gynecol Sci are open-access, distributed under the terms of the Creative Commons Attribution Non-Commercial License (http://creativecommons. org/licenses/by-nc/3.0/) which permits unrestricted non-commercial use, distribution, and reproduction in any medium, provided the original work is properly cited.

Copyright $\odot 2019$ Korean Society of Obstetrics and Gynecology 


\section{Obstetrics \& Gynecology Science}

Ayse Erdem Yayla, et al. Human papillomavirus in married women

developing countries than in developed countries [3].

Genotypes of HPV are divided into two categories-highrisk (HR-HPV) and low-risk (LR-HPV)-according to their ability to cause cancer [4]. LR-HPVs cause genital warts, and HRHPVs cause abnormal cellular changes which progress to cancer in the cervix, vulva, and anus.

Majority (up to $90 \%$ ) of newly acquired HPV infections similarly become undetectable within 1-2 years [5]. The HPV-DNA test is recommended for the detection of patients with infection in the latent period and that of HPV types. HPV-DNA detection is also necessary to produce effective vaccines. Women positive for the presence of HPV-DNA are considered to be infected and follow-up of HPV infections is based on HPV-DNA measurements at four, six, and twelvemonth intervals [6].

Intensive studies on these viruses and their genotypes are being performed worldwide. The identification of commonly found genotypes in both, risk-patient groups, and in the general population is important for the prevention of HPV infection and for follow-up of associated diseases. Also, phylogenetic analyses and determination of the similarity of the HPV strains isolated from different regions help to clarify how viral genes and species develop, and which strains should be used for developing vaccines.

A study reported that more than $85 \%$ of the new HPV cases and HPV deaths occur in low-middle income countries [7]. According to the Republic of Turkey Ministry of Health data for 2016, Northeastern Anatolia, which includes Erzurum, is the region with the highest proportion (42.7\%) of the rural population [8]. There are regional differences in terms of socio-cultural and economic levels of development in Turkey. Erzurum is located in a disadvantaged region due to high altitude of over 1,850 m and a terrestrial climate. Also, Erzurum shows poorer socio-economic development than the average of that seen in other parts of Turkey according to the development index [9]. In such a region, morbidity owing to HPV is expected to be high. Thus, we aimed to detect HPV-DNA positivity and to type and phylogenetically analyze isolated HPVs from smear samples taken from married women who visited the hospital for routine gynecological examination. Also, we wanted to report the first results for HPV genotypes for Turkey.

\section{Materials and methods}

\section{Study group}

This study was supported by the Atatürk University Scientific Research Projects Coordination Unit. The study included 263 married women who visited the Nene Hatun Maternity Hospital between April and December 2016 for routine gynecological examination. We selected cases from amongst women who had no history of malignancy. None of the women had a history of high-grade cervical intraepithelial neoplasia or cervical/vaginal/vulvar/anal cancer, or loop electrosurgical excision procedure conization treatment and hysterectomy. We provided detailed information to the patients about the procedure for collection of the cervical samples, and we provided them with questionnaires to complete in order to obtain their sociodemographic information and to determine their risk factors for HPV infection. Informed consent was obtained from all the women involved in the study.

\section{Collection of specimens}

Cervical smear specimens of patients applied to the hospital for gynecological examination were collected using a sterile speculum followed using a single-use sterile cervical brush which was rotated $360^{\circ}$ clockwise in the cervical os. Samples were transferred to the Atatürk University Medical Faculty Molecular Microbiology Laboratory in sterile tubes along with the brush. In the lab, $1 \mathrm{~mL}$ sterile saline was added to the samples, and they were centrifuged at 3,500 rpm for 15 minutes. Then, fluids containing samples were transferred into Eppendorf tubes and were stored at $-80^{\circ} \mathrm{C}$ in the freezer until DNA isolation was performed.

\section{DNA isolation}

A GF-1 Viral Nucleic Acid Extraction kit (Vivantis Technologies, Subang Jaya, Malaysia) was used to isolate viral DNA from cervical smear specimens. DNA isolation was performed according to the manufacturer's recommendations. First, 200 $\mu \mathrm{L}$ of the samples homogenized by vortexing were transferred to eppendorf tubes and $50 \mu \mathrm{L}$ proteinase $\mathrm{K}$ was added to them. Then, $215 \mu \mathrm{L}$ of Buffer VL containing Carrier RNA was added and mixed using pulsed-vortexing. This mixture was incubated at $65^{\circ} \mathrm{C}$ for 10 minutes in the heating block. At the end of the incubation, $280 \mu \mathrm{L}$ of absolute ethanol was added to each sample and samples were vortexed again. Samples transferred to filter tubes were centrifuged at 5,000 


\section{Obstetrics \& Gynecology Science}

Vol. 62, No. 4, 2019

$\times \mathrm{g}$ for 1 minute. Next, $500 \mu \mathrm{L}$ of wash buffer 1 solution was added and samples were centrifuged at 5,000 $\times \mathrm{g}$ for 1 minute. Wash buffer was added to the samples and they were centrifuged three times, twice for 1 minute each and once for 3 minutes at $5000 \times \mathrm{g}$. Lastly, they were centrifuged at 5,000 $\times \mathrm{g}$ for 1 minute. After each centrifugation, the remaining fraction was decanted. The nucleic acid suspensions thus obtained were maintained at $-20^{\circ} \mathrm{C}$ until the polymerase chain reaction (PCR) assay was performed.

\section{Polymerase chain reaction}

In order to detect HPV-DNA, a PCR assay for the L1 gene region was performed. For this, the MY09/MY11 primer pair was used for the first round and then the GP5/GP6 primer pair for the second-round. The PCR reaction was carried out under conditions specified by Entiauspe et al. [10]. The primer sequences, target gene region, and amplicon sizes used for the study are shown in Table 1.

The PCR mix used for the experiment was adjusted to a volume of $50 \mu \mathrm{L}$ per sample. The PCR mix included reagents in the following amounts, PCR Master Mix $25 \mu \mathrm{L}$ (Thermo scientific 2X PCR master mix, Life Technologies, Carlsbad, CA, USA); Forward primer (MY09/GP5) $1 \mu \mathrm{L}$; Reverse primer (MY11/GP6) $1 \mu \mathrm{L}$, ddH2O $17 \mu \mathrm{L}$, and $6 \mu \mathrm{L}$ of template DNA were added to the reaction mixture. The ABI Data (Applied Biosystems, Foster City, CA, USA) thermal cycler was used for PCR. Amplification using primers HPV MY09/11 was performed as follows: $95^{\circ} \mathrm{C}$ for 5 minutes, 40 cycles of $95^{\circ} \mathrm{C}$ for 1 minute, $55^{\circ} \mathrm{C}$ for 1 minute, $72^{\circ} \mathrm{C}$ for 1 minute, ending with $72^{\circ} \mathrm{C}$ for 10 minutes. Nested PCR was performed with the MY09/11 and GP5+/6+ primer sets. Amplification cycles were modified as follows: $95^{\circ} \mathrm{C} 5$ minutes, 40 cycles of $94^{\circ} \mathrm{C}$ 30 seconds, $45^{\circ} \mathrm{C} 30$ seconds, $72^{\circ} \mathrm{C} 30$ seconds, ending with $72^{\circ} \mathrm{C}$ for 10 minutes.

HPV-specific amplification products were visualized using a $1 \%$ agarose gel stained with ethidium bromide (Sigma-
Chemical Co., St. Louis, MO, USA). Image evaluation was performed using the ultraviolet imaging system (VilberLourmat, Collégien, France). The PCR-amplified HPV-positive amplicons were purified with the QIAquick PCR Purification kit (Qiagen, Hilden, Germany).

\section{Sequencing and phylogenetic analysis}

Sequence analysis was performed for forward and reverse sequences using purified HPV-positive DNA products and the MY09/MY11 primers. Sequence analysis was performed using the Sanger sequencing method and the BigDye Terminator v3.1 Cycle Sequence kit, using the ABI 3110xI DNA Analyzer (Applied Biosystems). Nucleotide sequences were evaluated using the Geospiza FinchTV program version 1.4.0 (Geospiza, Inc. Seattle, WA, USA). Our working samples and reference HPV-DNA sequences from the NCBI GenBank (http://www.ncbi.nlm.nih.gov/genbank/) were aligned using the BioEdit software package (v7.2.5).

\section{Statistical analysis}

Data are given in numbers and percentages. Statistical analyses were performed to determine the relationships between sociodemographic variables. The SPSS software (version 20.0, SPSS Inc., Chicago, IL, USA) was used for analyses. The $\chi^{2}$ test was used to determine statistical significance, $P<0.05$ was considered to be statistically significant.

\section{Results}

Voluntarily provided cervical smear specimens from 263 women who had no history of malignancy were investigated for the presence of HPV. Patients were provided with a questionnaire in order to obtain their socio-demographic information, in which they were required to provide their educational status, professional status, history of alcohol consumption,

Table 1. Primers used in human papillomavirus screening and typing

\begin{tabular}{lccc}
\hline Primer & Primer sequence (5'-3') & Target gen & Length (bp) \\
\hline MY09 & CGT CCM ARR GGA WAC TGA TC & L1 \\
MY11 & GCM CAG GGW CAT AAY AAT GG & \\
GP5+ & TTT GTT ACT GTG GTA GAT ACT AC & L1 \\
GP6+ & GAAAA TAA ACT GTA AAT CAT ATT C & 140 \\
& M=A+C, R=A+G, W=A+T, Y $=$ C + T & \\
\hline
\end{tabular}




\section{Obstetrics \& Gynecology Science}

Ayse Erdem Yayla, et al. Human papillomavirus in married women

and history of sexually transmitted congenital syphilis or any other sexually transmitted diseases (STDs). The data are summarized in Table 2.

The age group of the women enrolled for the study included women between 18-80 years, with an average age of 39 years, with a standard deviation of \pm 11.4 years. Majority of the cases belonged to the age group of 35-44 years; the highest HPV positivity was also seen in this group. HPV positivity increased rapidly from 18 to 44 years of age and decreased rapidly after the age of 45 . However, the distribution of HPV positivity in the age groups did not show a statistically significant difference $(P=0.66)$.

According to the results of the survey, seven women were employed, and the rest were housewives; except for one of the HPV positive women, the others were housewives. HPV positivity did not differ statistically according to the professional status of the subjects $(P=0.39)$.

Majority of the women (85.9\%) had completed educa- tion up to secondary school level or up to lower educational levels. The proportion of women with education from high school and above was only $14.1 \%$. However, no statistically significant difference was found between education levels and HPV positivity $(P=0.42)$.

None of the women had a history of alcohol consumption, thus, in our study, all HPV strains were isolated from women who did not consume alcohol. Thirty-five women (13.3\%) were found to be habituated to cigarette smoking (from 2 to 40 cigarettes per day). Three of the HPV-positive cases were smokers; however, HPV positivity did not differ statistically between smokers and nonsmokers ( $P=0.48$ ).

Two women had a history of STDs. One had a history of condyloma, whereas, the other woman's husband was affected with condyloma at the time of the study. However, the second woman was not included in the phylogenetic analysis, because sequence results from her samples were unclear. No statistically significant relationship was found be-

Table 2. Human papillomavirus (HPV) positivity in cases according to various demographic indications

\begin{tabular}{|c|c|c|c|c|}
\hline Sociodemographic variables & HPV-positive & HPV-negative & Total & $P$-value \\
\hline \multicolumn{5}{|l|}{ Mean age $(39.0 \pm 11.4)$} \\
\hline Age groups & & & & 0.66 \\
\hline $18-24$ & $1(5.9)$ & $25(10.2)$ & $26(9.9)$ & \\
\hline $25-34$ & $4(23.5)$ & $65(26.4)$ & $69(26.2)$ & \\
\hline $35-44$ & $9(52.9)$ & $89(36.2)$ & $98(37.3)$ & \\
\hline $45-60$ & $2(11.8)$ & $55(22.4)$ & $57(21.7)$ & \\
\hline$>60$ & $1(5.9)$ & $12(4.9)$ & $13(4.9)$ & \\
\hline Occupation & & & & 0.38 \\
\hline Working & $1(5.9)$ & $6(2.4)$ & $7(2.7)$ & \\
\hline Housewife & $16(94.1)$ & $240(97.6)$ & $256(97.3)$ & \\
\hline Education status & & & & 0.27 \\
\hline Middle school or less & $13(76.5)$ & $213(86.6)$ & $226(85.9)$ & \\
\hline High school and up & $4(23.5)$ & $33(13.4)$ & $37(14.1)$ & \\
\hline Alcohol use & & & & - \\
\hline No & $17(100.0)$ & $246(100.0)$ & $263(100.0)$ & \\
\hline Smoking & & & & 0.48 \\
\hline Yes & $3(17.6)$ & $32(13.0)$ & $35(13.3)$ & \\
\hline No & $14(82.4)$ & $214(87.0)$ & $228(86.7)$ & \\
\hline STD history & & & & 0.13 \\
\hline Yes & $1(5.9)$ & $1(5.9)$ & $2(0.8)$ & \\
\hline No & $16(94.1)$ & 245 (94.1) & $261(99.2)$ & \\
\hline
\end{tabular}

Values are presented as number (\%).

STD, sexually transmitted disease. 


\title{
Obstetrics \& Gynecology Science
}

\author{
Vol. 62, No. 4, 2019
}

tween the presence of STDs and HPV positivity.

Seventeen HPV-positive samples were sequenced. Phylogenetic analysis was performed for 14 , excluding 3 samples for which sequencing did not work well. Six of the typed samples were shown to be HPV 70 positive, four were HPV 16 positive, and the remaining four were HPV 54, 72, 81, and 114 positive. A total prevalence of $6.5 \%$ was found; of these, $1.5 \%$ were identified as HR-HPV and $3.8 \%$ as LRHPV. Distribution of HPV types according to risk groups is shown in Table 3.

Fig. 1 shows the phylogenetic analysis of the L1 gene region in the HPV-DNA of the strains included in the study. Five genera, including Alpha, Beta, Gamma, Mu, and $\mathrm{Nu}$, which cause human diseases, were included in the phylogenetic analysis. Reference genotypes obtained from the NCBI GenBank were used to construct the phylogenetic tree. The reference numbers of these strains were as follows: U45910, U45911, U45909, U45908, U45907, U45913, U45889, U44891, U45890, U45893, U45894, KT725857, KU298919, KU298928, EU918767, GQ244463, KC470249, KX514417, U21941, KU298914, HE574705, AJ620209, X94164, AF536179, HM596522, AF402678, AF472509, AF536180, DQ057331, DQ057332, DQ057330, DQ057327, KU298909, JX129488, M74117, KX514422, U45897, U45895, U45896, NC001457, X74464, NC001354, and U06714. Sequencing and phylogenetic analysis showed that all strains used for this study belonged to the genus Alphapapillomavirus affecting humans.

\section{Discussion}

It is reported that the prevalence of HPV infection and HPVinduced disorders in Turkey is lower than that in many other developed countries, however, in recent years, the rates have increasing [11]. Bruni et al. [12] determined worldwide HPV

Table 3. Distribution of human papillomavirus (HPV) types according to risk groups

\begin{tabular}{lcc}
\hline Risk group & HPV types & No. (\%) \\
\hline LR-HPV & $54,70,72,81,114$ & $10(3.8)$ \\
HR-HPV & 16 & $4(1.5)$ \\
Undefined HPV & - & $3(1.1)$ \\
Total & - & $17(6.5)$ \\
\hline
\end{tabular}

$H R$, high-risk; LR, low-risk. prevalence which was found to be $11.7 \%$ in a meta-analysis study. In a study conducted in Thailand by Phoolcharoen et al. [13], highest HPV prevalence was seen in women between 20-30 years of age. Martins et al. [14] observed that the frequency of HPV infection decreased with increasing age in Brazilian women. In the multicenter study conducted in Turkey by Dursun et al. [15], highest HPV prevalence was detected in women under the age of 25, followed by that in the age group of 30-39 years, and then in women above 60 years of age. In our study, highest positivity of HPV-DNA was found in the 35-44 age group (9.2\%) and the lowest positivity was found under 25 years (3.8\%). These results are in contrast to those from other studies. It is not possible to comment on the low prevalence in the under- 25 group, because the subjects were not asked to specify their age at the first instance of coitus and/or at the time of marriage for this study. However, we believe that the low HPV-DNA positivity in women under 25 years of age is probably related to the sexual habits of the women and their husbands, none of them have a history of malignancy and their lifestyles are modest.

Information about the prevalence of HPV obtained from a low-income population has been presented in this study, however, contrary to our expectation, the prevalence of HPV is low in Erzurum. Subjects enrolled for the study come from a conservative background, and they follow customs and are accustomed to reserved environment. Loyalty on part of both spouses is an essential part of our culture r. In addition, contraction of STDs is considered as dishonorable by the people of this region and hence, privacy of such cases poses an obstacle to sexual relations with multiple partners. Such a lifestyle and socio-cultural structure substantially prevents the risk of transmission of HPV. Men from this culture are circumcised according to tradition. Circumcision probably reduces HPV prevalence in Erzurum as well as in other provinces of Turkey. None of the subjects enrolled for the study are alcohol or substance addicts. We would like to emphasize that the most important factor in the low HPV prevalence found in our study is the absence of harmful urinogenital diseases in women.

The distribution of common HPV genotypes in the world varies from country to country. For example, the three most prevalent genotypes are 16,31, and 6, found in Southern Italy [16]; 16, 51, and 52 in Thailand [13]; 58, 16, and 52 in Korea [17]; 53, 58, and 52 in Korea as found in a later study 


\section{Obstetrics \& Gynecology Science}

Ayse Erdem Yayla, et al. Human papillomavirus in married women

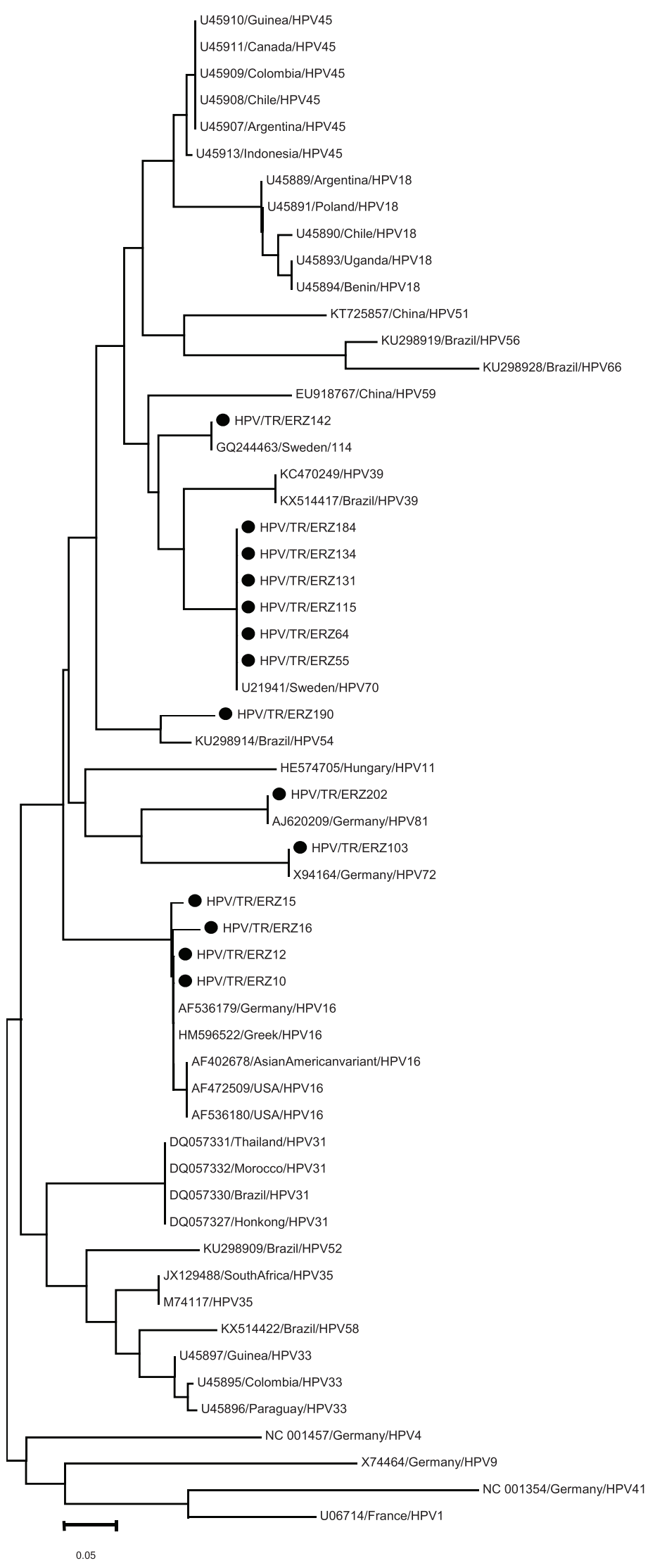

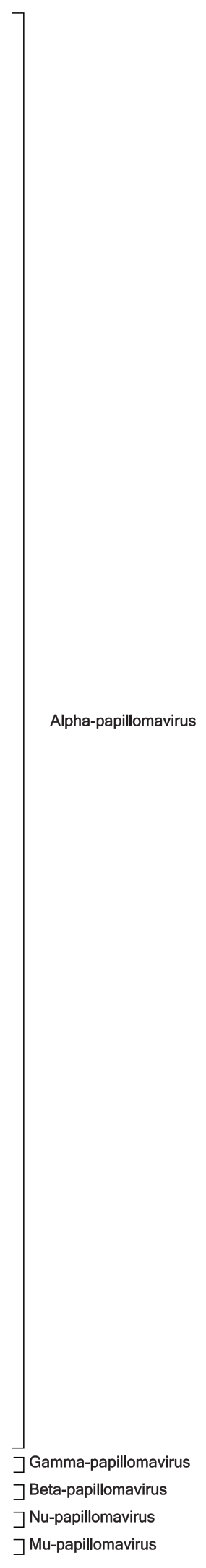

Fig. 1. Phylogenetic analysis of the $\mathrm{L} 1$ gene region in human papillomaviruses (HPV)-DNA positive specimens. HPVs classified in five different genera are indicated in the phylogenetic tree. All of our study samples in which the Alpha genus was included are shown with rounded black dots. Other examples mentioned in the phylogenetic tree were obtained from the National Center for Biotechnology Information (NCBI) GenBank and are shown by the access numbers. 


\title{
Obstetrics \& Gynecology Science
}

\author{
Vol. 62, No. 4, 2019
}

[18]; and 51, 11, and 16 in Qatar [19]. The most prevalent genotypes in provinces from different regions of Turkey are 16, 45, and 18 in Adana [20]; 18 and 16 in Konya [21]; 16, 11, and 6 in Ankara [22]; 16, 56, and 51 in Antalya [23]; 16, 18, and 33 in Elazig [24]; and 16, 18, 31, and 51 in Istanbul [25]. In our study, the most frequently seen genotypes were 70 and 16. Only one genotype was isolated among other genotypes including 54, 72, 81, and 114 found in our study. All other genotypes isolated in the study, apart from HPV 70, are frequently isolated in different regions of Turkey as well as in other countries. In this study, 1.5\% of the identified genotypes were HR-HPV and the remaining (3.8\%) were LRHPV. Naturally, in our study, low-risk HPVs were detected at a higher prevalence than high-risk HPVs because subjects included in the study consisted of women who did not have malignant conditions such as high-grade cervical intraepithelial neoplasia, cervical/vaginal/vulvar/anal cancer.

HPV 70/85 is considered to be the HPV type of the highest mucosal oncogenic viral subdivision. Due to the close phylogenetic relationship with known carcinogenic types, this type has been introduced into the International Agency for Research on Cancer (IARC) category 2B, the category that includes carcinogens that affect humans [26]. HPV 70 is a genotype that can be isolated not only from women but also from genital warts of men [27]. We want to draw attention to the fact that this type of HPV has been reported for the first time in Turkey. HPV 81, 54 and 72 genotypes isolated in our study were also isolated in another study from Italy by Vergori et al. [28], from anal swab specimens of HIV-positive men. HPV114 was isolated from a low-grade cervical lesion by Ekström et al. [29] in Sweden.

It is most likely that, the differences in results obtained from our study according to the general profile of Turkey depends on the test method used, the number of samples, and preferences in sexual behavior of people living in the region. Although there are various studies that involve the identification and typing of HPV-DNA in cervical smear samples in the country, no large-scale study has been conducted for phylogenetic analysis of HPV. Phylogenetic analysis revealed the kinship grades of the strains obtained in our province using data from NCBI GenBank. The sequences of ERZ184, -134, $-131,-115,-64$ and -55 strains isolated in our study clustered with the Swedish HPV70 strain. ERZ25, -16, -12 and -10 isolated from this study clustered with the European (Greek and Germany) HPV16 strains, however, the Asian American variant and the American HPV16 strains clustered in a separate group. The strains ERZ190, -103, -202 and -142 isolated from our study clustered with strains from HPV 54 (Brazilian strain), HPV 72 (German strain), HPV 81 (German strain) and HPV 114 (Swedish strain), respectively.

Recently, a number of studies have been performed to determine phylogeny and nucleotide variability of multiple or single HPV types. In one of these studies, Aziz and colleagues found HPV 6, 55, 11, 43, 33, 18, 16 types in Pakistani women, and $80-99 \%$ nucleotide identity with types related to the same species of HPV. They reported that the sequences clustered with those from China, India, Mexico, Iran, Slovenia and Germany [30]. In China, Chen et al. [31] have isolated HPV 26, 51, 69, 82, 30, 53, 56, 66, 34, 73, 54 and 61, and they reported that heterogeneity and phylogeny of HPV isolates show an independent evolutionary history for each species. Cui et al. [32] reported that the E6 and E7 genes show nucleotide polymorphisms in some of the high-risk HPV45 strains identified in Southwest China. Conde-Ferraez et al. [33] reported that the E7 oncogene from the HPV type 58 was more conserved than the E6 gene in women from Southeast Mexico, and HPV 58 variants were not specific to a geographic region. Ramas et al. [34] reported that HPV16 variants isolated from Uruguayan women mostly clustered in the European lineage and a small part of them clustered in the Asian-American/North-American and African lineage, and additionally identified new variants in the European and African clusters. In China, Zhang et al. [35] identified mutation sites in the E5, E6, E7 and L1 genes of a significant proportion HPV 31 strains. These studies have shown that the distribution of HPV types in different countries is not affected to a great extent by geographical distances. Also, it emphasizes the importance of nucleotide polymorphisms and mutational changes in viral genes. In general, the magnitude of the prevalence of European HPV types in different countries, including Turkey, is noteworthy. So far, only one study has been conducted on the phylogenetic analysis of HPV genotypes in Turkey. In this study, Avci et al. [22] have performed a phylogenetic analysis of HPV 16, 11 and 6 detected in women with cervical pathologies from Ankara. However, it was not possible to compare our findings with this study as the details of phylogenetic characterization were not provided.

In conclusion, this study is the first study showing phylogenetic analysis and molecular characterization of circulating HPV types in married women over 18 years of age from the 


\section{Obstetrics \& Gynecology Science}

Ayse Erdem Yayla, et al. Human papillomavirus in married women

Eastern Anatolia Region of Turkey. In order to contribute to vaccine studies and to enrich the global HPV data, it is necessary to perform a greater number of such studies with larger sample sizes. The reason for the low rate of HR-HPV in our study is probably the fact that the subjects include women without malignant conditions. It is possible to say that their traditional and conservative lifestyle also contributes to the low rate of HR-HPV. Nonetheless, 1.5\% women representing the normal population are at a risk of encountering HR-HPV strains.

\section{Conflict of interest}

No potential conflict of interest relevant to this article was reported.

\section{Ethical approval}

Ethical approval for this study was obtained from the Atatürk University Medical Faculty Institutional Ethics Committees (Approval No. B.30.2.ATA.0.01.00/50). Required permissions for the collection of clinical specimens were obtained from the provincial public health directorate of Erzurum.

\section{Patient consent}

Informed consent was obtained from all the women involved in the study.

\section{References}

1. Abramowitz L, Lacau Saint Guily J, Moyal-Barracco M, Bergeron C, Borne H, Dahlab A, et al. Epidemiological and economic burden of potentially HPV-related cancers in France. PLoS One 2018;13:e0202564.

2. Chow LT, Broker TR, Steinberg BM. The natural history of human papillomavirus infections of the mucosal epithelia. APMIS 2010;118:422-49.

3. Thun MJ, DeLancey JO, Center MM, Jemal A, Ward EM. The global burden of cancer: priorities for prevention. Carcinogenesis 2010;31:100-10.
4. Ceccarelli M, Rullo EV, Facciolà A, Madeddu G, Cacopardo $B$, Taibi $R$, et al. Head and neck squamous cell carcinoma and its correlation with human papillomavirus in people living with HIV: a systematic review. Oncotarget 2018;9:17171-80.

5. Schiffman M, Castle PE, Jeronimo J, Rodriguez AC, Wacholder S. Human papillomavirus and cervical cancer. Lancet 2007;370:890-907.

6. Rompalo A. Preventing sexually transmitted infections: back to basics. J Clin Invest 2011;121:4580-3.

7. Sahasrabuddhe VV, Parham GP, Mwanahamuntu MH, Vermund SH. Cervical cancer prevention in low- and middle-income countries: feasible, affordable, essential. Cancer Prev Res (Phila) 2012;5:11-7.

8. Republic of Turkey Ministry of Health. Health Statistics Yearbook 2016 [Internet]. Ankara (TR): General Directorate of Health Research; c2018 [cited 2018 Oct 21]. Available from: https://dosyasb.saglik.gov.tr/ Eklenti/13160,sy2016enpdf.pdf?0.

9. Tulan D, Türko ES. Women entrepreneurship: a survey in Erzurum province. Finans Ekon Sos Araşt Derg 2018;3:285-306.

10. Entiauspe L, Nunes E, Collares T, da Silveira MF, Seixas F. Comparison between two methods for molecular characterization of human papillomavirus. J Bras Doenças Sex Transm 2013;25:13-5.

11. Ozgul N. Genital warts. Turkiye Klinikleri J Gynecol Obstet Spec Top 2017;10:174-80.

12. Bruni L, Diaz M, Castellsagué $X$, Ferrer E, Bosch FX, de Sanjosé $\mathrm{S}$. Cervical human papillomavirus prevalence in 5 continents: meta-analysis of 1 million women with normal cytological findings. J Infect Dis 2010;202:178999.

13. Phoolcharoen N, Kantathavorn N, Sricharunrat $T$, Saeloo $\mathrm{S}$, Krongthong W. A population-based study of cervical cytology findings and human papillomavirus infection in a suburban area of Thailand. Gynecol Oncol Rep 2017:21:73-7.

14. Martins TR, Mendes de Oliveira C, Rosa LR, de Campos Centrone $\mathrm{C}$, Rodrigues $\mathrm{CL}$, Villa $\mathrm{LL}$, et al. HPV genotype distribution in Brazilian women with and without cervical lesions: correlation to cytological data. Virol J 2016;13:138.

15. Dursun $P$, Ayhan A, Mutlu L, Çağlar M, Haberal A, Güngör T, et al. HPV types in Turkey: multicenter hos- 


\section{Obstetrics \& Gynecology Science}

Vol. 62, No. 4, 2019

pital based evaluation of 6388 patients in Turkish gynecologic oncology group centers. Turk Patoloji Derg 2013;29:210-6.

16. Coscia MF, Monno R, Ballini A, Mirgaldi R, Dipalma G, Pettini $F$, et al. Human papilloma virus (HPV) genotypes prevalence in a region of South Italy (Apulia). Ann Ist Super Sanita 2015;51:248-51.

17. Kim MJ, Kim JJ, Kim S. Type-specific prevalence of highrisk human papillomavirus by cervical cytology and age: data from the health check-ups of 7,014 Korean women. Obstet Gynecol Sci 2013;56:110-20.

18. Ouh YT, Min KJ, Cho HW, Ki M, Oh JK, Shin SY, et al. Prevalence of human papillomavirus genotypes and precancerous cervical lesions in a screening population in the Republic of Korea, 2014-2016. J Gynecol Oncol 2018;29:e14.

19. Bansal D, Elmi AA, Skariah S, Haddad P, Abu-Raddad LJ, Al Hamadi AH, et al. Molecular epidemiology and genotype distribution of Human Papillomavirus (HPV) among Arab women in the State of Qatar. J Transl Med 2014; 12:300.

20. Altun Z, Yarkin F, Vardar MA, Uguz AH. The prevalence of human papilloma virus infection among women who admitted to Cukurova University Faculty of Medicine Hospital. Turkiye Klinikleri J Med Sci 2011;31:307-14.

21. Findik D, Dagi HT, Arslan U, Findik Y. Frequency and genotype distribution of human papillomavirus in cervical specimens. Genel Tıp Derg 2012;22:116-20.

22. Avci GA, Bozdayi G, Taskiran Ç, Ozkan S, Onan MA. Phylogenetic analysis and prevalence of human papillomavirus (HPV) in women with cervical pathologies. J Turk Soc Obstet Gynecol 2013;10:151-9.

23. Unal B, Sezer C. Analysis of high risk HPV subtypes associated with cervical intraepithelial neoplasia: a single centre retrospective study in the Mediterranean region of Turkey. Turk Patoloji Derg 2014;30:85-6.

24. Bulut $Y$, Belhan M, Özercan IH. Detection of human papillomavirus genotypes by pyrosequencing method in uterine cervical cancer samples. Firat Univ Med J Health Sci 2016;30:71-5.

25. Barışık NO, Keser SH, Gül AE, Şimşek EE, Özdemir HG. Prevalence of high-risk human papilloma virus and identification of type using real-time polymerase chain reaction analysis and liquid-based cytology. South Clin Istanb Eurasia 2017;28:175-80.
26. Giuliodori K, Campanati A, Liberati G, Ganzetti G, Giangiacomi M, Marinelli K, et al. Lifelong widespread warts associated with human papillomavirus type 70/85: a new diagnostic entity? Acta Dermatovenerol Alp Panonica Adriat 2016;25:11-3.

27. Potocnik M, Kocjan B, Seme K, Poljak M. Distribution of human papillomavirus (HPV) genotypes in genital warts from males in Slovenia. Acta Dermatovenerol Alp Panonica Adriat 2007;16:91-6.

28. Vergori A, Garbuglia AR, Piselli P, Del Nonno F, Sias C, Lupi $F$, et al. Oral human papillomavirus DNA detection in HIV-positive men: prevalence, predictors, and cooccurrence at anal site. BMC Infect Dis 2018;18:25.

29. Ekström J, Forslund O, Dillner J. Three novel papillomaviruses (HPV109, HPV112 and HPV114) and their presence in cutaneous and mucosal samples. Virology 2010;397:331-6.

30. Aziz H, Iqbal H, Mahmood H, Fatima S, Faheem M, Sat-

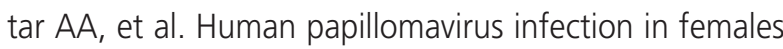
with normal cervical cytology: genotyping and phylogenetic analysis among women in Punjab, Pakistan. Int J Infect Dis 2018;66:83-9.

31. Chen Z, Schiffman M, Herrero R, DeSalle R, Anastos K, Segondy $M$, et al. Classification and evolution of human papillomavirus genome variants: alpha-5 (HPV26, 51, 69, 82), alpha-6 (HPV30, 53, 56, 66), alpha-11 (HPV34, 73), alpha-13 (HPV54) and alpha-3 (HPV61). Virology 2018;516:86-101.

32. Cui F, Zhang Z, Xu J, Ding X, Mu X, Wan Q, et al. Genetic variability and lineage phylogeny of human papillomavirus type 45 based on E6 and E7 genes in Southwest China. Virus Res 2018;255:85-9.

33. Conde-Ferraez L, Pacheco-Arjona R, Novelo Canul C, Gomez-Carballo J, Ramirez-Prado JH, Ayora-Talavera G, et al. Genetic variability in E6 and E7 oncogenes from human papillomavirus type 58 in Mexican women. Intervirology 2017;60:235-46.

34. Ramas V, Mirazo S, Bonilla S, Ruchansky D, Arbiza J. Analysis of human papillomavirus 16 E6, E7 genes and Long Control Region in cervical samples from Uruguayan women. Gene 2018;654:103-9.

35. Zhang J, Zhang S, Wang M, Ding X, Wen Q, Chen Z, et al. Genetic variability in E5, E6, E7 and L1 genes of human papillomavirus type 31. Mol Med Rep 2018;17:5498-507. 